Claudia Wirthlin*

\title{
Bücher für den Frieden
}

\author{
Eine Initiative der Fachbibliothek Mission 21 - nicht nur für Südsudan
}

https://doi.org/10.1515/bfp-2021-0077

Zusammenfassung: Die Idee zur Unterstützung von zwei Institutsbibliotheken im Südsudan entstand 2018 im Gespräch mit dem Leiter der Presbyterianischen Kirche im Lesesaal der Fachbibliothek Mission 21 in Basel. Es ging dabei um die wichtige Rolle von Bildung und kritischer Auseinandersetzung mit der eigenen Geschichte beim Aufbau einer friedlichen Zukunft des kriegsversehrten Landes. Nicht zuletzt profitierte auch die Bibliothek in Basel in verschiedenster Hinsicht von der friedensfördernden Initiative.

Schlüsselwörter: Mission 21; Südsudan; Frieden; Agenda 2030

\section{Books for Peace}

An Initiative of the Specialised Library Mission 21 - Not Only for South Sudan

Abstract: The idea of supporting two libraries in South Sudan arose while meeting the president of the Presbyterian Church of South Sudan 2018 in Basel. Sitting in the lecture room of the Mission 21 Specialised Library, the conversation turned around the importance of higher education for a peaceful future of the country and the urgency of reflecting critically on one's own history. In the end, our library in Basel could also benefit in many ways from our peace promoting initiative.

Keywords: Mission 21; South Sudan; peace; Agenda 2030

\section{Einleitung}

Die Fachbibliothek der Nichtregierungsorganisation Mission $21^{1}$ in Basel ist eng verflochten mit der 1815 gegründeten Basler Mission², einer protestantischen Missionsgesellschaft, die seit 2001 - zusammen mit zwei kleineren

1 Mission 21 (2021b).

2 Basler Mission (2021).

*Kontaktperson: Claudia Wirthlin, claudia.wirthlin@sunrise.ch
Missionsgesellschaften - unter dem Namen Mission $21^{3}$ auftritt. In enger Verbindung mit dem weltweit bekannten Forschungsarchiv ${ }^{4}$ blickt die Bibliothek auf eine gut zweihundertjährige Sammeltätigkeit zurück.

Im Verlauf der Jahre fanden - parallel zu den zeitgenössischen Schwerpunkten in der Projekt- und Programmarbeit von Mission 21 (bis 2000: der Basler Mission) immer wieder neue Themen Eingang in die Bibliotheksbestände. Zwei Beispiele dafür aus der neueren Zeit sind die Aufnahme des Themenschwerpunkts „Entwicklungspolitik“ seit den 1960er-Jahren und der gezielte Ausbau des Schwerpunktes „African Studies“ ab 2001.

Die circa 35000 Einheiten (Monografien und Zeitschriften) umfassende Bibliothek ist eine One Person Library und steht nicht nur einer breiten Öffentlichkeit und Studierenden offen, sondern ihr Angebot war immer auch auf die Bedürfnisse der hier Beschäftigten ausgerichtet. ${ }^{5}$ So stand sie etwa den Länder- und Projektverantwortlichen bei Literaturbeschaffung und Spezialrecherchen zur Seite und informierte sie regelmäßig über neuerschienene Publikationen in ihrem Fachgebiet. Fachspezifische Beratungen waren nicht nur bei Studierenden oder anderen Interessierten beliebt und geschätzt, sondern auch bei Mitarbeitenden und Gästen aus dem nationalen und internationalen Netzwerk von Mission 21.

\section{Frieden, Gerechtigkeit und inklusive Gesellschaften: der Beitrag der Bibliothek}

Die seit 2017 auch in der Schweiz verstärkt geführte Diskussion rund um die 17 Ziele für nachhaltige Entwicklung (Sustainable Development Goals, SDGs) der Vereinten Na-

3 Mission 21 (2021a).

4 Mission 21 (2021c).

5 Leider wurde die Bibliotheksstelle nach meiner Pensionierung Ende Juli 2020 coronabedingt nicht wieder besetzt. Deshalb der sprachliche Wechsel ins Präteritum. Meine Aktivitäten im Rahmen der Agenda 2030 beziehen sich auf den Zeitraum 2017-2019. Die Bibliothek funktioniert seit August 2020 nur noch eingeschränkt, die OnlineAusleihe wird nun vom Archivteam betreut. 
tionen führte zu einer Neuausrichtung der Projekt- und Programmarbeit auch bei Mission 21. Zusammen mit weiteren Organisationen aus dem reformierten Umfeld der Schweiz setzt Mission 21 den Schwerpunkt auf fragile und konfliktbetroffene Gebiete. ${ }^{6}$ In enger Anlehnung an den Ansatz „SDG 16 Plus“ der „Pathfinders for Peaceful, Just and Inclusive Societies " ${ }^{\text {"7 }}$ hat sie sich Frieden, Gerechtigkeit und inklusive Gesellschaften auf die Fahnen geschrieben.

Die Umsetzung dieser Themen in der Programmarbeit beschäftigte nicht nur Mission 21 als glaubensbasierte Organisation insgesamt, sondern auch die Bibliothek setzte sich intensiv damit auseinander und wurde in folgenden drei Bereichen aktiv:

- Fachliteratur in der Bibliothek: Ab 2017 wurde das Angebot an Fachliteratur zu den verschiedenen Aspekten der SDGs gezielt erweitert, wobei insbesondere die Frage nach dem Beitrag der Religionen zu einem nachhaltigen Frieden in verschiedenen Kontexten weltweit - etwa im Südsudan, in Kamerun oder in Indonesien - berücksichtigt wurde.

- Sensibilisierung intern und extern: Die Bibliothek bemühte sich, die SDGs intern und extern bekannter zu machen: Anfang 2019 mit einer kleinen Ausstellung im Eingangsbereich zur Bibliothek und einem Hausvortrag für alle Mitarbeitenden. Im Frühling konnte ich die Verbundbibliotheken auf dem Platz Basel zum Thema „Bibliotheken und Agenda 2030“ sensibilisieren. Zudem wies ich in verschiedenen Artikeln auf die Thematik hin, so etwa in der Online-Publikation „à propos“ von Swiss Peace. ${ }^{8}$ Das nötige Rüstzeug für diese und andere Aktivitäten holte ich mir im Sommer 2018 in einer Weiterbildung an der Hochschule der Medien in Stuttgart. ${ }^{9}$

- Bücher für den Südsudan: Diese Initiative entstand spontan und hatte zum Ziel, zwei unterdotierten Bibliotheken im kriegsversehrten Südsudan Bücher zu spenden. Beide Bibliotheken gehören zu Bildungsinstituten, die von der Presbyterianischen Kirche des Südsudan PCOSS,,$^{10}$ Partnerorganisation von Mission 21, getragen werden. Sie sind Teil des Kooperationsprogrammes Südsudan, ${ }^{11}$ das auf das Oberziel Frieden und Versöhnung ausgerichtet ist.

6 Kooperationsgemeinschaft KOGE (2021).

7 Pathfinders for Peaceful, Just and Inclusive Societies (2021).

8 Wirthlin (2019a), Wirthlin (2019b).

9 Die „LIS International Summer School 2018“ umfasste u.a. den Workshop „Lobbying and Advocacy: How to promote libraries by changing the world in partnership“, der mir sehr zustatten kam.

10 Mission 21 (2021g).

11 Mission 21 (2021e).

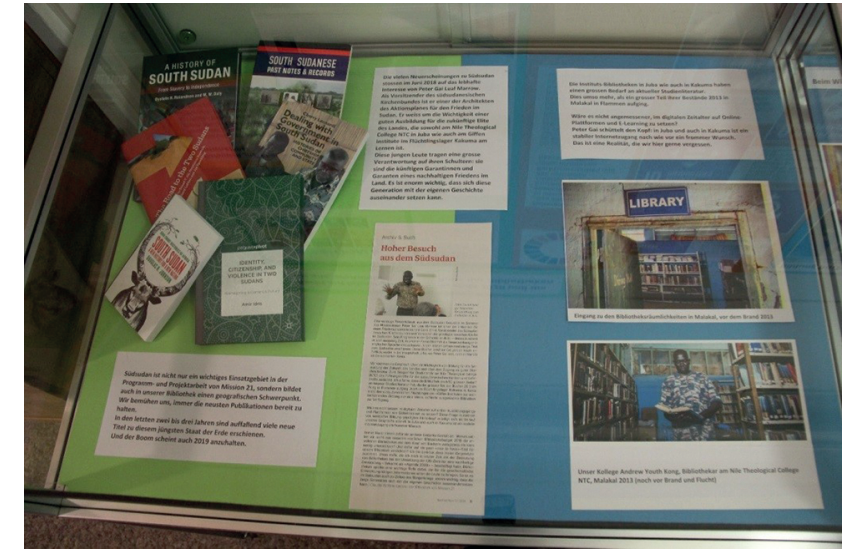

Abb. 1: Blick in eine Ausstellungsvitrine im Eingangsbereich zur Bibliothek Mission 21, @Mission 21

Im Folgenden gehe ich näher auf diese dritte Bibliotheksaktivität ein. Ich zeige auf, wie es zu diesem Projekt überhaupt kam, welche Ziele damit verfolgt wurden und inwiefern Bücher im spezifischen Kontext des Südsudan eine friedensfördernde Wirkung haben können.

\section{Bücher für Südsudan}

\subsection{Die Lage im Südsudan}

Der jüngste Staat der Welt wurde 2011 nach langjährigen Kämpfen unabhängig. Bereits Ende 2013 brach jedoch erneut Krieg aus, zunächst im Nordosten. Im Juli 2016 weitete sich der Konflikt auf das ganze Land aus, 2018 wurde ein Friedensvertrag geschlossen und 2020 eine Einheitsregierung gebildet. Die Situation ist heute nach wie vor äußerst fragil und unstabil. Staatliche Strukturen sind extrem schwach, Bildungs- und Gesundheitseinrichtungen zerstört. Die Kirchen, vereint unter dem Dach des Südsudanesischen Kirchenbundes SSCC, ${ }^{12}$ sind in dieser Situation auf allen Gebieten wichtige Akteure beim Wiederaufbau des Landes, genießen zudem als einzige Institutionen das Vertrauen der Bevölkerung und sind im ganzen Land nah bei den Menschen.

Über 400000 Tote forderte der Bürgerkrieg seit 2013 und bei einer Gesamtbevölkerung von 11 Millionen leben heute noch gut 4 Millionen Geflüchtete in Lagern in- und außerhalb der Landesgrenzen. ${ }^{13}$

12 Mission 21 (2021h).

13 Es ist schwierig, verlässliche Zahlen anzugeben. Hier nur eine von verschiedenen Quellen: Statistisches Bundesamt (2020). 


\subsection{Hoher Besuch in Basel und eine spontane Idee}

Als Ende Mai 2018 Peter Gai Lual Marrow, Präsident der PCOSS und einer der Architekten des Friedensabkommens, im Basler Missionshaus zu Gast weilte, nahm er sich trotz dichter Agenda Zeit, im Lesesaal der Bibliothek die englischsprachigen Neuerwerbungen zu Südsudan durchzusehen.

Gerade hatte ich den Buchbestand zum Schwerpunkt Südsudan erneuert, denn in den Jahren 2014-2018 war aus aktuellem Anlass eine ganze Menge neuer Titel erschienen, die meisten in kleiner Auflage und bei renommierten Verlagen.

Wir kamen ins Gespräch: über die Wichtigkeit von Bildung für die Gestaltung der Zukunft des Landes, über die Schäden, die der Bürgerkrieg in der Bevölkerung angerichtet hatte. Ganze Generationen von jungen Menschen wuchsen ohne jegliche Schulbildung heran. ${ }^{14}$ Qualitativ hochwertige Bildung sei aber der Schlüssel zum künftigen Frieden im Land, meinte Peter Gai. Deshalb engagiere sich seine Kirche in der Ausbildung neuer Führungskräfte (Pfarrpersonen und Lehrkräfte), welche später - zurück in den Gemeinden - ihr neuerworbenes Wissen dafür einsetzten, gesellschaftliche Gräben zu kitten und alltägliche Konflikte mit friedlichen Mitteln zu lösen. Es sei enorm wichtig, dass die junge Generation lerne, sich mit der eigenen Geschichte kritisch auseinanderzusetzen.

Spätestens an dieser Stelle begann ich fieberhaft zu überlegen, was ich als Bibliothekarin tun könnte, um die Bibliotheken am „Nile Theological College NTC“"15 in Juba und am „Giffen Institute of Theology GIT“16 im Flüchtlingslager Kakuma zu unterstützen. Die Bestände beider Bibliotheken waren 2013 beim Ausbruch des Bürgerkriegs am alten Standort größtenteils in Flammen aufgegangen und ab 2015 in Juba und Kakuma in Nordkenia erst teilweise wieder aufgebaut worden.

Während des Gesprächs mit Peter Gai fing mein Bibliothekarinnenherz Feuer und schon am nächsten Tag nahm - vorerst in meinem Kopf - ein kleines Projekt Gestalt an. Warum sollten Studierende in den Bibliotheken des NTC und GIT nicht genauso auf aktuellste Fachliteratur zugreifen können wie Studierende in Basel? Und heißt es nicht in Zielvorgabe 4.3 der Agenda 2030, ${ }^{17}$ dass Männer und Frauen überall gleichermaßen Zugang auch zu höhe-

14 65,5 \% der Erwachsenen können weder lesen noch schreiben, UNDP (2020).

15 Mission 21 (2021f).

16 Mission 21 (2021d).

$17 \mathrm{UN}$ (2021a). rer Bildung haben sollen? Und in Unterziel 16.10, alle müssten öffentlichen Zugang zu Information haben ${ }^{18}$

\subsection{Gedacht, getan: zur Umsetzung der Projektidee}

Nach Rücksprache mit der Programmverantwortlichen für Südsudan bei Mission 21, vertieften Diskussionen mit Peter Gai und grünem Licht meiner Vorgesetzten konnte ich für beide Bibliotheken aus dem laufenden Bibliotheksbudget noch im Sommer 2018 je 21 englischsprachige Fachbücher einkaufen. Die Werke erreichten bis Anfang 2019 im Handgepäck von Vertrauenspersonen portionsweise ihre Bestimmungsorte. Im Herbst 2019 kamen nochmals je fünf neuerschienene Titel dazu und zusätzlich 20 Titel für die Kirchenleitung am Hauptsitz in Juba. Die Trefferliste aus der Schweizer Rechercheplattform Swisscovery zum Begriff „South Sudan“, gefiltert nach Bibliothek Mission 21, gibt einen Überblick über die thematische Bandbreite der Fachbücher. ${ }^{19}$ Insgesamt wurden auf diese Weise 72 Bücher im Wert von knapp 6000 CHF transferiert.

Bei den Vorabklärungen zu dieser Initiative machte ich mir ein möglichst genaues Bild über die Situation der beiden Institute und ihrer Bibliotheken. Insbesondere klärte ich ab, ob es im digitalen Zeitalter überhaupt noch zeitgemäß war, im Sektor höhere Bildung auf Bücher zu setzen. Schnell war klar, dass ein extrem unstabiler Internetzugang und die mangelhafte Ausstattung der Studierenden mit Computern und Tablets die Unterstützung mit Online-Fachliteratur nicht zuließen. Bücher zu schicken machte also durchaus Sinn, selbst wenn es nur wenige waren. Besser ein Tropfen auf den heißen Stein als gar nichts tun, dachte ich.

\section{Initiative statt Projekt: eine Bilanz}

Allzu großen Aufwand konnte ich angesichts des eingeschränkten Umfangs des Projekts nicht betreiben. Ein ordentlicher Projektbericht oder gar eine professionelle Evaluation lagen aus personellen und zeitlichen Gründen außerhalb des Machbaren. Ich zog und ziehe es deshalb auch vor, von Initiative oder Aktion zu sprechen statt von Projekt.

$18 \mathrm{UN}(2021 b)$

19 Wirthlin (2021) 


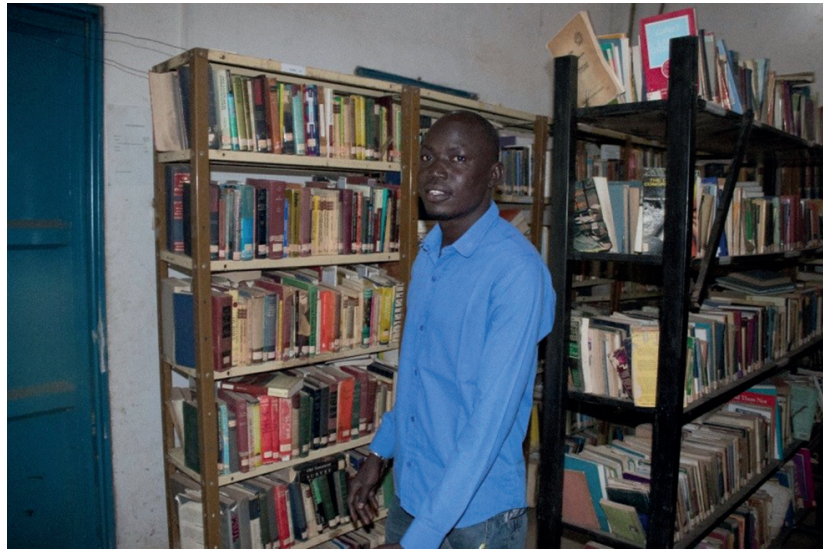

Abb. 2: Studentischer Mitarbeiter in der Bibliothek des Nile Theological Center NTC in Juba im Oktober 2018, (CSilvano Yokwe/ Copyright Mission 21

Wie stark Studierende, Lehrpersonen und die Kirchenleitung von meiner Initiative profitieren konnten, lässt sich nicht mit Zahlen belegen. Wir wissen nicht, wie viele Personen die Bücher tatsächlich in Händen hielten oder gar lasen. Vielleicht konnten die 89 Studienanfänger am NTC - davon 10 Frauen - bereits vom Bücherzuwachs in der Bibliothek profitieren? Oder vielleicht die Lehrpersonen beim Vorbereiten der Kurse? Oder die Mitglieder der Kirchenleitung beim Ausarbeiten von Konfliktlösungsstrategien?

Die Pandemie macht eine nachträgliche Beurteilung zusätzlich schwierig. Die Bibliotheken blieben während der verschiedenen Lockdown-Phasen meist geschlossen. Auch mit dem Internet gab und gibt es nach wie vor Probleme. Obwohl z.B. das NTC Ende 2020 größere und ITmäßig besser ausgestattete Räume beziehen konnte, war der Internetzugang nicht stabil genug, um den Unterricht wegen der Coronapandemie ganz auf Online-Kurse umzustellen. ${ }^{20}$ Gerade kürzlich berichtete der Institutsdirektor von erneuten Schwierigkeiten: Während des neuerlichen Lockdowns würden die Studierenden schriftliche Kursunterlagen und Hausaufgaben persönlich in der Schule abholen und alle Interaktionen zwischen Lehrpersonen und Studierenden fänden via WhatsApp statt. ${ }^{21}$

20 Am GIT im Lager Kakuma hingegen konnten 2020 die meisten Kurse während des Lockdowns in Kenia online durchgeführt werden. Die Studierenden konnten auch vom neuen, der Bibliothek angeschlossenen Computerraum für Recherchen profitieren.

21 Die E-Mail vom 23.03.21 von Rev. Santino Odong Othol an Dorina Waldmeyer, verantwortlich bei Mission 21 für die Programmarbeit in Südsudan, liegt mir vor.

\section{Fazit}

Insgesamt hat sich die Initiative trotz mangelnder Reichweite und Professionalität für mich persönlich gelohnt, denn der Blick über den eigenen Tellerrand hinaus war motivierend und sinnstiftend. Ich konnte so die eigene Arbeit in einen größeren Zusammenhang stellen.

Und, last but not least: die Initiative bot mir die perfekte Gelegenheit, inner- und außerhalb von Mission 21 auf die Dienstleistungen der eigenen Fachbibliothek aufmerksam zu machen. Aus dem Hintergrund hervortreten, hinstehen und laut sagen, was wir Bibliotheken alles leisten, dazu bietet die Agenda 2030 den perfekten Rahmen. Eine Erfahrung, die ich meinen Berufskolleginnen und -kollegen wärmstens empfehlen kann.

\section{Literaturverzeichnis}

Basler Mission (2021): Basler Mission. Verfügbar unter https://www. baselmission.org/.

Kooperationsgemeinschaft KOGE (2021): Kooperationsgemeinschaft KOGE. Verfügbar unter https://koge.ch/public/news.

Mission 21 (2021a): Mission 21. Verfügbar unter https://www.mis sion-21.org/.

Mission 21 (2021b): Bibliothek. Verfügbar unter https://www.mis sion-21.org/was-wir-tun/forschung/bibliothek.

Mission 21 (2021c): Forschungsarchiv. Verfügbar unter https://www. mission-21.org/was-wir-tun/forschung.

Mission 21 (2021d): GIT, Giffen Institute of Theology. Verfügbar unter https://www.mission-21.org/was-wir-tun/projekte-und-part ner/projekt/erwachsenenbildung-fuer-die-gestaltung-der-zu kunft.

Mission 21 (2021e): Kooperationsprogramm Südsudan. Verfügbar unter https://www.mission-21.org/was-wir-tun/projekte-und-p artner/projekt/kooperationsprogramm-suedsudan.

Mission 21 (2021f): NTC, Nile Theological College. Verfügbar unter htt ps://www.mission-21.org/was-wir-tun/projekte-und-partner/pr ojekt/religion-als-mittel-fuer-frieden-und-entwicklung-im-sued sudan.

Mission 21 (2021g): PCOSS, Presbyterianische Kirche des Südsudan. Verfügbar unter https://www.mission-21.org/was-wir-tun/pro jekte-und-partner/partner/pcoss-presbyterianische-kirche-dessuedsudan.

Mission 21 (2021h): SSCC, Südsudanesischer Kirchenbund. Verfügbar unter https://www.mission-21.org/was-wir-tun/projek te-und-partner/partner/sscc-suedsudanesischer-kirchen bund.

Pathfinders for Peaceful, Just and Inclusive Societies (2021): Pathfinders for Peaceful, Just and Inclusive Societies. Verfügbar unter https://www.sdg16.plus.

Statistisches Bundesamt Destatis (2020): Statistisches Bundesamt Destatis. Südsudan, statistisches Länderprofil. Verfügbar unter https://www.destatis.de/DE/Themen/Laender-Regionen/In ternationales/Laenderprofile/sued-sudan.pdf?_blob=publicati onFile. 
UN (2021a): United Nations, Department of Economic and Social Affairs. Sustainable development goals, 4. Verfügbar unter https://sdgs.un.org/goals/goal4.

UN (2021b): United Nations, Department of Economic and Social Affairs. Sustainable development goals, 16. Verfügbar unter https://sdgs.un.org/goals/goal16.

UNDP (2020): United Nations Development Programme. Human Development Reports: South Sudan, human development indicators: education. Verfügbar unter http://www.hdr.undp.org/en/.

Wirthlin, Claudia (2019a): Bibliotheken weltweit leisten einen Beitrag zum Frieden. In: à propos, 161 (Juli). Verfügbar unter https://ww w.swisspeace.ch/apropos/bibliotheken-weltweit-leisten-einenbeitrag-zum-frieden/?lang=de.

Wirthlin, Claudia (2019b): Libraries worldwide make a contribution to peace. In: à propos, 161 (July). Verfügbar unter https://www.swi sspeace.ch/apropos/bibliotheken-weltweit-leisten-einen-bei trag-zum-frieden/.

Wirthlin, Claudia (2021): Trefferliste aus Swisscovery Basel. Verfügbar unter https://basel.swisscovery.org/.

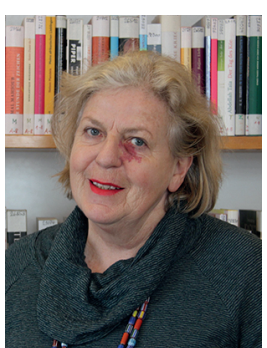

\section{Claudia Wirthlin}

Leiterin A.D. der Fachbibliothek Mission 21

in Basel

Neubadstrasse 126

CH-4054 Basel

Schweiz

claudia.wirthlin@sunrise.ch 